\title{
EFFECT OF SPECIMEN SIZE ON THE VARIANT SELECTION IN MARTENSITIC TRANSFORMATION
}

\author{
HIROFUMI MIYAJI and EI-ICHI FURUBAYASHI \\ Materials Characterization Division, National Research Institute for Metals, \\ 2-3-12, Nakameguro, Meguro-ku, Tokyo 153 Japan
}

(Received November 5, 1989)

\begin{abstract}
In thin sheet the anisotropy of the internal stress due to constraint by the surrounding parent phase which will be abbreviated as "constraint stress," seems to occur during the martensitic transformation and the variant selection is expected also in the transformation without external stress. To verify this, the textures were measured by X-ray pole figure method on the $\gamma$ and $\alpha$ phase which were developed in thin sheet of $\mathrm{Fe}-30 \mathrm{Ni}$ alloy prepared by severe cold rolling and annealing followed by subzero quenching to liquid Nitrogen. Experimental data were analyzed with conventional variant selection models. The results obtained are as follows: (a) Variant selection phenomenon was clearly observed and the results could be explained with conventional variant selection models. (b) It is expected that different results occur between the conventional variant selection models when the martensitic transformation occurs accompanying the strain components of $\left|\varepsilon_{1}\right| \leqq\left|\varepsilon_{2}\right|$, where $\varepsilon_{1}$ is the strain along the Bain compression axis and $\varepsilon_{2}$ is along the perpendicular axis.
\end{abstract}

KEY WORDS Martensitic transformation, variant selection, Bain relation, transformation texture, iron nickel alloy, constraint stress.

\section{INTRODUCTION}

In the martensitic transformation of ferrous materials, it is well known that the variant of martensite phase is selected, when the external stress is applied during transformation. This is observed by the experiments to apply such as tensile or compressive stress by the universal testing machine (Bokrov and Parker, 1963; Higo, Lecroisey and Mori, 1974; Kato and Mori, 1976; Kato and Mori, 1977; Kato, Monzen and Mori, 1978; Sato et al., 1980), or rolling stress by the rolling mill (Kubotera, Nakaoka and Nagamine, 1966; Abe, Ito and Fukumoto, 1967; Abe and Ito, 1967; Borik and Richman, 1967; Watanabe, Araki and Miyaji, 1971; Stone and Thomas, 1974; Yutori and Ogawa, 1979; Hashimoto, Satoh and Tanaka, 1980). Some models concerning the appearence mechanism of variant selection phenomenon have been proposed on the basis of these experimental results (Patel and Cohen, 1953; Bokrov and Parker, 1963; Bogers and Burgers, 1964; Kubotera, Nakaoka and Nagamine, 1966; Abe and Ito, 1967; Borik and Richman, 1967; Olsen and Jesser, 1971; Higo, Lecroisey and Mori, 1974; Kato and Mori, 1977).

Among them, the important models which seem to explain the experimental results satisfactorily, were proposed recently. At first, Higo, Lecroisey and Mori (1974) pointed out the importance of the initial stage of the transformation from 


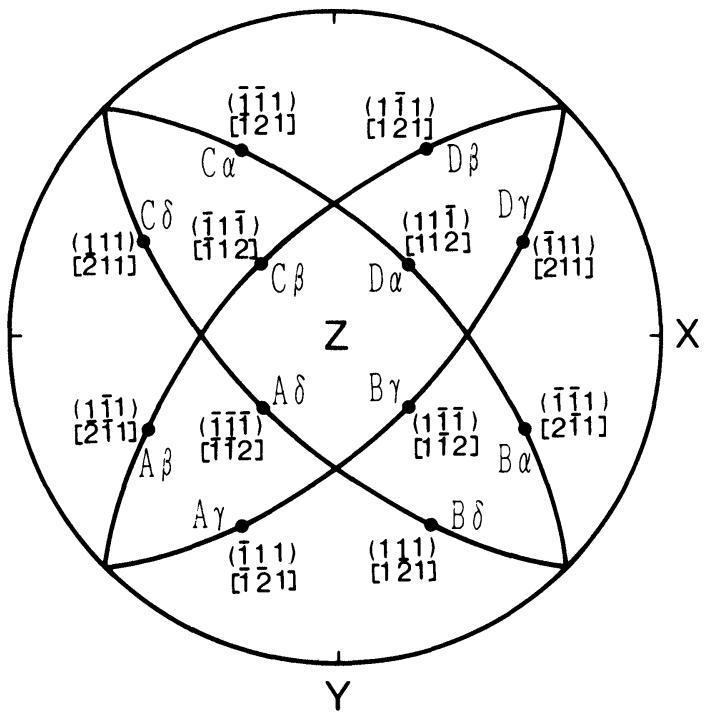

Figure 1 Stereographic projections of the $\{111\}_{\gamma}\langle 112\rangle_{\gamma}$ shear systems together with the symbols of the Thompson tetrahedron.

the parent phase to the martensite phase which occurred by the shear deformation of lattice of the small amount of participated atoms. On the transformation mechanism of martensite, the models which are composed of combination of some elementary processes in which one or two shear deformation is concerned, were proposed by Kurdjumov and Sachs $(1930)$, Nishiyama $(1934 ; 1936)$ and Bogers and Burgers (1964). All of them postulate that the shear movement of atoms on (111) plane to $\langle 112\rangle$ direction occurs at the beginning of transformation. This shear deformation (strain) is defined as the characteristic strain in this concept. Poles of $(111)\langle 112\rangle$ shear system can be plotted on the standard stereographic projections with the symbols described according to Thompson's tetrahedron notation method, as shown in Figure 1. Y phase possesses 12 twin shear systems in all and Higo, Lecroisey and Mori (1974) showed that the variants induced by the active system, which has the maximum resolved shear stress and is assisted more effectively by the applied stress, occur predominantly. This concept will be called "Twinning Shear (TS) model," since the (111) $\langle 112\rangle$ shear coincides with the twinning shear in FCC metals. On the other hand, Furubayashi (1985a; 1985b) and Furubayashi, Miyaji and Nobuki (1987) remarked to the interaction between the stress and "lattice deformation" in the phenomenological theory of martensitic transformation. Austenite lattice (face centered cubic structure) may also be described as a body centered tetragonal structure with an axial ratio of $\sqrt{2}$ as seen in Figure 2. Bain (1924) proposed that the martensitic transformation merely involves the deformation of about $20 \%$ compression to the $C$ axis ([001] direction) and about $12 \%$ expansion to the direction perpendicular to it of the austenite unit cell, as shown in Figure 2. This deformation (strain) is defined as the characteristic strain in this concept. There are three ways of choosing this tetragonal lattice in the austenite lattice, that is, the direction of $C$ axis can be taken parallel to either one of $X, Y$, or $Z$ axis respectively and Furubayashi 


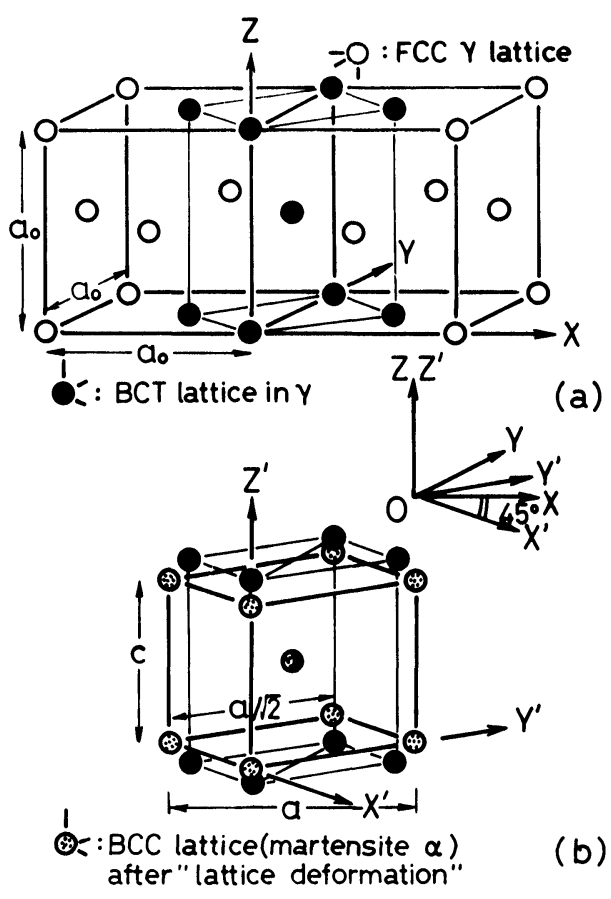

Figure 2 (a) Lattice correspondence and (b) Bain strain associated with martensitic transformation from $\gamma$ to $\alpha$.

(1985a; 1985b) considered that such a variant as the deformation accompanied by martensitic transformation is assisted most effectively by the applied stress (the work done by the stress is maximum), is chosen. This view will be called "Bain Strain (BS) model" hereafter. The BS model differs from the TS model by Higo, Lecroisey and Mori (1974) who looked at the easiness of shear deformation which occurs in the beginning of Bain deformation and satisfies the K-S, Nishiyama and Bogers-Burgers mechanism. The attractive point to use Bain relation is that the rotation axis of it is no other than the Bain compression axis of the variant. This makes one to get exact knowledge of variant selection due to the applied stress. The orientation deviation of Bain variants from $\mathrm{K}-\mathrm{S}$ or $\mathrm{N}-\mathrm{W}$ variants is about $11.1 \mathrm{deg}$ and $9.7 \mathrm{deg}$, respectively. This will not be too large to misunderstanding the exact variant selection rule, because different variants from a $\gamma$ crystal have much larger orientation difference e.g. of $62.8 \mathrm{deg}$ between Bain variants. Before these works, Hashimoto, Satoh and Tanaka (1980) showed that the variant selection phenomenon occurs in thin sheet of steel even without external stress, because the heterogeneity of internal stress due to constraint by the surrounding parent phase which will be abbreviated as "constraint stress" makes an effect at the martensitic transformation. They thought that the constraint stress due to transformation strain would be weaker in the normal direction of the sheet than in other directions.

But the experimental confirmation of the variant selection phenomenon was not conducted in Hashimoto's study. Because they used low carbon steel having 
high transformation temperature, it is impossible to get crystallographic information of the parent phase, as it is lost at room temperature. The constraint stress always prevents the strain accompanied by the transformation, which differs in the case of the external stress. To study these differences seems to lead to some important results of the variant selection phenomenon.

Then, the present authors conducted the experimental study to find whether or not the variant selection phenomenon would appear in the martensitic transformation in thin sheet of $\mathrm{Fe}-30 \mathrm{Ni}$ alloys even though the external stress is not applied. Both parent and transformed phase were obtained at room temperature because the Ms temperature of the alloy lay near the room temperature. The variant selection could be determined by texture analysis. The discussion was also made to the results whether they can be explained by the variant selection rules already proposed.

In addition, Hashimoto and Furubayashi are based on the same standpoint as to the assistance of lattice deformation of the martensite variant by stress or strain. But Furubayashi's model seems much more simple than Hashimoto's model to understand intuitively the interaction between atom displacement and stress in martensitic transformation, and also to compare the relationship with the model of Higo, Lecroisey and Mori (1974).

\section{EXPERIMENTAL PROCEDURE}

As already mentioned, it is necessary to prepare the specimens satisfying following conditions for analyzing variant selection phenomenon by means of conventional X-ray pole figure method:

(1) Ms temperature has to be near the room temperature so as to get both the transformation and parent phases at room temperature.

(2) Simple texture must be developed in the parent $\gamma$ phase. Texture of the parent phase is desirable to have (001) orientation. The reason will be understood in the following sections.

Fe-30 Ni alloy was used as the specimens satisfying above conditions. Table 1 shows chemical composition and characteristic temperatures of transformation or recrystallization of the alloy used. Raw materials of electrolytic iron and electrolytic nickel were used to prepare the sample of $3.5 \mathrm{~kg}$ ingot by melting in an induction vacuum furnace. The specimens were prepared by heating the ingot at $1100^{\circ} \mathrm{C}$ for $1 \mathrm{~h}$, and hot rolling to $12 \mathrm{~mm}$ thickness and the surface layers were ground to the final thickness of $10 \mathrm{~mm}$. Then, to develop well the (001)[100] type texture (cube texture) in the parent phase, it was cold rolled by $96 \%$, and annealed at $800^{\circ} \mathrm{C}$ for $15 \mathrm{~min}$ for recrystallization according to Grewen and

Table 1 Chemical composition and transformation or recrystallization temperatures.

\begin{tabular}{c|ccccccc|ccccc}
\hline \multirow{2}{*}{ Alloy } & \multicolumn{7}{|c|}{ Composition (wt \%) } & \multicolumn{4}{c}{ Characteristic Temperature $\left({ }^{\circ} \mathrm{C}\right)$} \\
& $\mathrm{Ni}$ & $\mathrm{Mn}$ & $\mathrm{Al}$ & $\mathrm{C}$ & $\mathrm{N}$ & $\mathrm{S1}$ & $\mathrm{S}$ & Af & Ms & $\mathrm{Md}$ & $\mathrm{Tr}$ \\
\hline $30 \mathrm{Ni}$ & 29.67 & 0.085 & 0.025 & 0.001 & 0.001 & 0.002 & 0.003 & 490 & -10 & $\sim 140$ & $700<$ \\
\hline
\end{tabular}


Wassermann (1975). It was chemically thinned to $40 \mu \mathrm{m}$ thin sheet sample containing the central layer of rolled sheet, and subzero-quenched to liquid nitrogen to induce martensitic transformation.

The variant selection phenomenon was analyzed by observing the texture during the transformation. (001) pole figures of austenite and martensite were determined by X-ray Schulz (reflection) or Decker (transmission) method, using Co target. Pole densities were shown by relative X-ray intensity with respect to a random sample and are indicated by arabic numerals in the pole figures.

\section{EXPERIMENTAL RESULT AND DISCUSSION}

(001) pole figure of austenite is shown in Figure 3. Austenite has well developed cube texture. (001) pole figure of martensite is shown in Figure 4. (001) pole figure of hypothetical martensite phase containing all variants is schematically shown in Figure 5, which are transformed from (001)[100] standard oriented $\gamma$ phase crystal according to Bain relation.

By comparing these figures, it is seen that only one component of the three variants appeared in Figure 4. It is proved in this way that the variant selection happens in the martensitic transformation in the thin sheet. The variant formed in this condition is $Z$ component, that is, $C$ axis (compression axis) in Bain deformation is oriented to the normal direction of the sheet (see Figure 2). As already mentioned, in the variant selection rules, "Twinning Shear (TS) model" and "Bain Strain (BS) model" have been proved successfully in explaining the experimental results (Higo, Lecroisey and Mori, 1974; Furubayashi, 1985a; Furubayashi, 1985b; Furubayashi, Miyaji and Nobuki, 1987). If these models are postulated to be correct in the present study, a certain (external or internal) compressive stress, which assists the deformation associated with martensitic

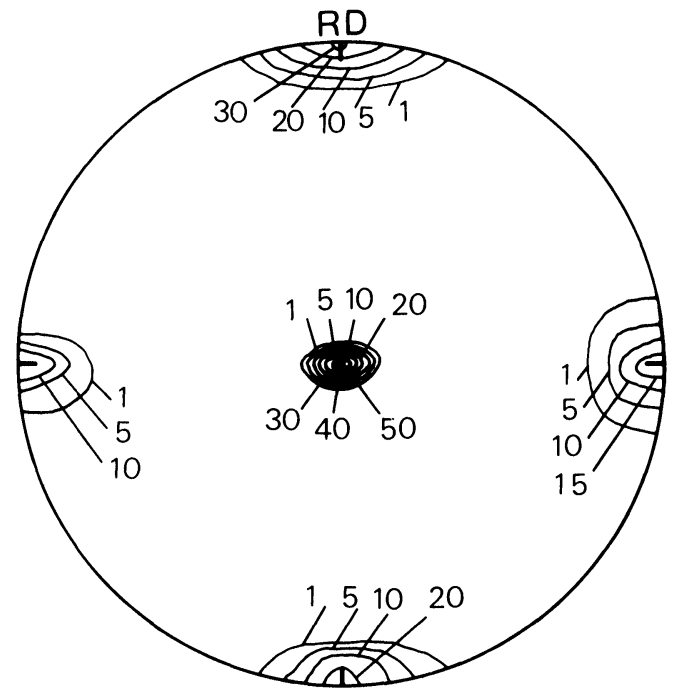

Figure 3 (001) pole figure of cube-textured austenite before transformation. 


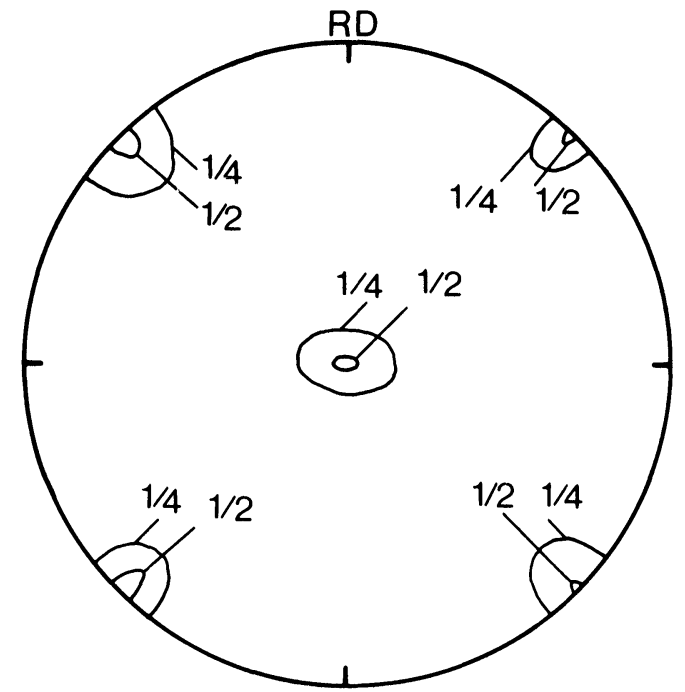

Figure 4 (001) pole figure of martensite transformed from cube-textured austenite.

transformation, must be applied to the normal direction of the sheet during the transformation. But in the present study, no external stress was applied, but only internal stress due to constraint would act in the specimen.

When the martensite phase forms in the parent phase, the constraint stress from the surroundings should act against the deformation associated with martensitic transformation at least in the initial stage of transformation, as pointed out by Hashimoto, Satoh and Tanaka (1980). In the case of thin sheet

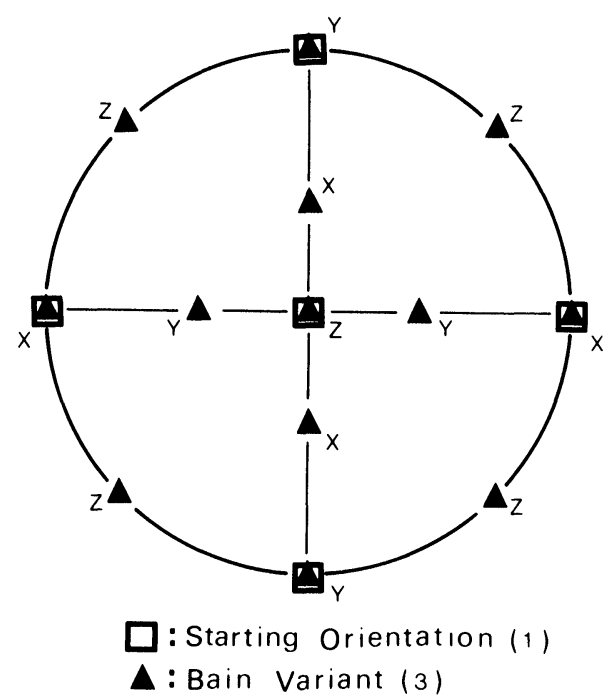

Figure 5 Schematic pole figure of all variants of $\alpha$ orientation, which are transformed from (001)[100] $\gamma$ orientation according to Bain relation. 
this constraint stress along the directions in the sheet plane will be the same as that of bulk sample, but will be negligibly small in the normal direction of the sheet.

Characteristic strain in BS model may be described in the following way. Let an austenite lattice (lattice parameter $a_{0}$ ) be transformed into a tetragonal lattice (c and $a)$ as a result of Bain deformation. The characteristic strain, $\varepsilon_{1}$ along the Bain compression axis and that $\varepsilon_{2}$ along the perpendicular axes will be described by Eqs. (1) and (2).

$$
\begin{aligned}
& \varepsilon_{1}=\left(c-a_{0}\right) / a_{0} \\
& \varepsilon_{2}=\left\{a-\left(a_{0} / \sqrt{2}\right)\right\} /\left(a_{0} / \sqrt{2}\right)
\end{aligned}
$$

Now, according to BS model, when the constraint stress along the normal direction of the sheet diminishes by $\Delta \sigma_{N}$, the work $W$, done by it decreases by

$$
\Delta W=\Delta \sigma_{N} \cos \theta \cdot \varepsilon_{1}+\Delta \sigma_{N} \sin \theta \cdot \varepsilon_{2}
$$

where $\theta$ is the angle between the Bain compression axis and the normal stress direction of the sheet, and $\Delta \sigma_{N}$ is positive to the compressive strain and negative to the expansive strain. The value of $\Delta W$ was $0.2 \Delta \sigma_{N}$ in variant $Z$ and $0.12 \Delta \sigma_{N}$ in variant $X$ and variant $Y$, respectively. Accordingly, variant $Z$ is most operative during the transformation.

On the other hand, according to TS model, the work $W$, done by the constraint stress decreases by

$$
\Delta W=\Delta \sigma_{N} \gamma_{t} \cos \phi \cos \lambda
$$

when the constraint stress along the normal direction of the sheet diminishes by $\Delta \sigma_{N}$, where $\gamma_{t}$ is the characteristic strain in TS model, $\phi$ is the angle between sheet plane and shear plane and $\lambda$ is the angle between the normal stress direction of $\Delta \sigma_{N}$ and the shear direction.

Schmid factors of the $\{111\}\langle 112\rangle$ shear systems for the [001] normal stress direction of the sheet is tabulated in Table 2 . Among them the four components $(A \delta, A \alpha, C \beta, B \gamma)$ have the largest value of 0.47 , and are most operative in view of the decrease of constraint stress along the normal direction of the sheet. It is evident from the crystallographical consideration of the martensitic transformation that the variant $Z$ in Bain lattice deformation occurs when these shear systems are operative, as shown in Figure 6 , in which the shear direction of these active shear systems are equally in the nearest direction to Bain compression axis of the variant $Z$ among three variants.

Accordingly the same results are derived from the variant selection rules of "Twinning Shear model" and "Bain Strain model." It is due to the fact that the martensitic transformation occurs satisfying $\left|\varepsilon_{1}\right|>\left|\varepsilon_{2}\right|$ in Eqs. (1) and (2) in the present study (Fe-30 Ni alloy). But the situation differs in other crystal systems: It is expected that different results would occur between these models when the martensitic transformation occurs accompanying the strain components of $\left|\varepsilon_{1}\right| \leqq$ $\left|\varepsilon_{2}\right|$ in Eqs. (1) and (2).

\section{CONCLUSIONS}

(1) Variant selection phenomenon was confirmed by texture determination for the martensite in thin sheets of $\mathrm{Fe}-30 \mathrm{Ni}$ alloy as obtained by quenching the cube-textured $\gamma$ phase. 
Table 2 Schmid factors for the $\{111\}_{\gamma}\langle 112\rangle_{\gamma}$ shear systems in the [001] normal direction of the sheet. Symbols are taken after the Thompson tetrahedron.

\begin{tabular}{|c|c|c|c|}
\hline Shear plane & Shear direction & Schmid factor & Symbol \\
\hline$\left(\begin{array}{lll}1 & 1 & 1\end{array}\right)$ & {$\left[\begin{array}{llll}\overline{2} & 1 & 1\end{array}\right]$} & 0.236 & $c \delta$ \\
\hline$\left(\begin{array}{lll}1 & 1 & 1\end{array}\right)$ & {$\left[\begin{array}{llll}1 & 2 & 1\end{array}\right]$} & 0.236 & $B \delta$ \\
\hline$\left(\begin{array}{lll}\overline{1} & \overline{1} & \overline{1}\end{array}\right)$ & {$\left[\begin{array}{llll}\overline{1} & \overline{1} & 2\end{array}\right]$} & -0.471 & A \\
\hline$\left(\begin{array}{lll}\overline{1} & \overline{1} & 1\end{array}\right)$ & {$\left[\begin{array}{llll}\overline{1} & 2 & 1\end{array}\right]$} & 0.236 & $C \propto x$ \\
\hline$\left(\begin{array}{lll}1 & 1 & 1\end{array}\right)$ & {$\left[\begin{array}{llll}2 & \overline{1} & 1\end{array}\right]$} & 0.236 & $\mathrm{~B} \alpha$ \\
\hline$\left(\begin{array}{lll}1 & 1 & 1\end{array}\right)$ & {$\left[\begin{array}{llll}1 & 1 & 2 & \end{array}\right]$} & -0.471 & $D \alpha$ \\
\hline$\left(\begin{array}{lll}1 & \overline{1} & 1\end{array}\right)$ & {$\left[\begin{array}{llll}1 & 2 & 1\end{array}\right]$} & 0.236 & $D B$ \\
\hline$\left(\begin{array}{lll}1 & \overline{1} & 1\end{array}\right)$ & {$\left[\begin{array}{llll}\overline{2} & \overline{1} & 1\end{array}\right]$} & 0.236 & $A \beta$ \\
\hline$\left(\begin{array}{lll}\overline{1} & 1 & \overline{1}\end{array}\right)$ & {$\left[\begin{array}{llll}\overline{1} & 1 & 2\end{array}\right]$} & -0.471 & $C B$ \\
\hline$\left(\begin{array}{lll}\overline{1} & 1 & 1\end{array}\right)$ & {$\left[\begin{array}{llll}2 & 1 & 1\end{array}\right]$} & 0.236 & $D \gamma$ \\
\hline$\left(\begin{array}{lll}1 & 1 & 1\end{array}\right)$ & {$\left[\begin{array}{llll}\overline{1} & \overline{2} & 1\end{array}\right]$} & 0.236 & $A \gamma$ \\
\hline$\left(\begin{array}{lll}1 & \overline{1} & \overline{1}\end{array}\right)$ & {$\left[\begin{array}{llll}1 & \overline{1} & 2\end{array}\right]$} & -0.471 & $\mathrm{~B} \gamma$ \\
\hline
\end{tabular}

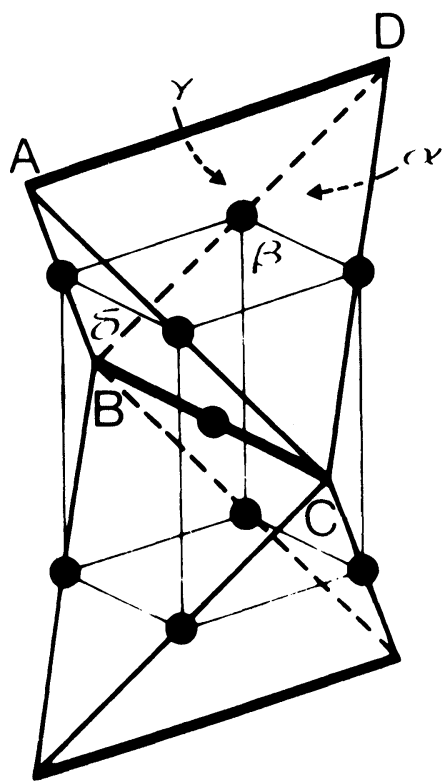

Figure 6 Tetragonal body centered cell in the f.c.c. lattice marked in Figure 2(a) and 2(b) is indicated together with two Thompson tetrahedrons. 
(2) Above results can be explained clearly with both "Twinning Shear (TS) model" and "Bain Strain (BS) model", if it is postulated that the component of constraint stress from the surrounding $\gamma$ phase falls only in the normal direction of the sheet during the transformation.

(3) It is expected that different results occur between these models when the martensitic transformation occurs accompanying the strain components of $\left|\varepsilon_{1}\right| \leqq$ $\left|\varepsilon_{2}\right|$, where $\varepsilon_{1}$ is the strain along the Bain compression axis and $\varepsilon_{2}$ is along the perpendicular axis.

\section{References}

Abe, H., Ito, K. and Fukumoto, R. (1967). On the Sheet-Texture of Fe-30.2 wt\% Ni Alloy. J. Jpn. Inst. Met. 31, 37-42.

Abe, H. and Ito, K. (1967). Effect of Nickel Content on the Texture of Martensite in Fe-Ni Alloy Rolled above $M_{d}$ Temperature. J. Jpn. Inst. Met. 31, 1300-1305.

Bain, E. C. (1924). Trans. AIME 70, 25.

Bokros, J. C. and Parker, E. R. (1963). The Mechanism of the Marteniste Burst Transformation in Fe-Ni Single Crystals. Acta Metall. 11, 1291-1301.

Bogers, A. J. and Burgers, W. G. (1964). Partial Dislocations on the $\{110\}$ Planes in the B. C. C. Lattice and the Transition of the F. C. C. into the B. C. C. Lattice. Acta Metall. 12, 255-261.

Borik, F. and Richman, R. H. (1967). Preferred Transformation in Strain-Hardened Austenite. Trans. Metall. Soc. AIME 239, 675-680.

Furubayashi, E. (1985a). Computer Simulation of Transformation Textures in Steels. Tetsu-toHagané 71, 1155-1162.

Furubayashi, E. (1985b). Transformation Texture Analysis with Bain Relation-Monte Carlo Simulations. Tetsu-to-Hagané 71, 1359-1366.

Furubayashi, E., Miyaji, H. and Nobuki, M. (1987). A Simple Model of Predicting the Transformation Textures in Thermomechanically Processed Steels. Trans. ISIJ 27, 513-519.

Grewen, J. and Wassermann, G. (1975). Oriented Nucleation in the Reverse Transformation of Fe-30 Ni. Texture 2, 45-56.

Higo, Y., Lecroisey, F. and Mori, T. (1974). Relation Between Applied Stress and Orientation Relationship of $\alpha^{\prime}$ Martensite in Stainless Steel Single Crystals. Acta Metall. 22, 313-323.

Hashimoto, O., Satoh, S. and Tanaka, T. (1980). Formation of $\alpha \rightarrow \gamma \rightarrow \alpha$ Transformation Texture in Sheet Steel. Tetsu-to-Hagané 66, 102-111.

Kurdjumov, G. and Sachs, G. (1930) Z. Phys. 64, 325.

Kubotera, H., Nakaoka, K. and Nagamine, T. (1966). Hot Rolling Texture of Low Carbon Steel. Tetsu-to-Hagané 52, 1171-1179.

Kato, M. and Mori, T. (1976). Stress-Induced Martensite in Single Crystals of an Fe-23Ni-5Cr Alloy. Acta Metall. 24, 853-860.

Kato, M. and Mori, T. (1977). Orientation of Martensite Formed in Fe-23Ni-5Cr Crystals under Uniaxial Stress Along [001]. Acta Metall. 25, 951-956.

Kato, M., Monzen, R. and Mori, T. (1978). A Stress-Induced Martensitic Transformation of Spherical Iron Particles in a Cu-Fe Alloy. Acta Metall. 26, 605-613.

Nishiyama, Z. (1934). Sci. Rep. Tohoku Univ. 23, 637.

Nishiyama, Z. (1936). Sci. Rep. Tohoku Univ. 25, 79.

Olsen, G. H. and Jesser, W. A. (1971). The Effect of Applied Stress on the F.C.C.-B.C.C. Transformation in Thin Iron Films. Acta Metall. 19, 1299-1302.

Patel, J. R. and Cohen, M. (1953). Criterion for the Action of Applied Stress in the Martensitic Transformation. Acta Metall. 1, 531-538.

Stone, G. and Thomas, G. (1974). Deformation Induced Alpha and Epsilon Martensites in Fe-Ni-Cr Single Crystals. Metall. Trans. 5, 2095-2102.

Sato, A., Kato, M., Sunaga, Y., Miyazaki, T. and Mori, T. (1980). Stress Induced Martensitic Transformation in Fe-Ni-C Alloy Single Crystals. Acta Metall. 28, 367-376.

Watanabe, S., Araki, T. and Miyaji, H. (1971). Strengthening Mechanism in Ausformed Steels. Proc. of International Conference on the Science and Technology of Iron and Steel, Suppl. to Trans. ISIJ 11, 1020-1025.

Yutori, T. and Ogawa, R. (1979). Formation of Transformation Texture in Controlled Rolled Steels. Tetsu-to-Hagané 65, 1747-1755. 